\title{
Modified SPAN-100 index in patients with intracerebral hemorrhage: Correlation and outcomes
}

\author{
Cyrus G. Escabillas', Kathreen Jane A. Lara ${ }^{2}$ and Jose C. Navarro ${ }^{3}$ \\ ${ }^{1}$ Resident, Department of Neurology, Jose R. Reyes Memorial Medical Center, Philippines \\ ${ }^{2}$ Neurovascular and Stroke Fellow, Department of Neurology, Jose R. Reyes Memorial Medical Center, Philippines \\ ${ }^{3}$ Consultant, Department of Neurology, Jose R. Reyes Memorial Medical Center, Philippines
}

\section{Introduction}

Cerebrovascular disease is the $2^{\text {nd }}$ most common disease in the world and is one of the leading cause of long-term disability [1]. Primary intracerebral hemorrhage accounts for approximately $10-15 \%$ of all type of stroke and is considered to have the highest mortality rate [2]. Survivors of intracerebral hemorrhage increases the risk of multiple chronic condition including hypertension, diabetes and cardiac problems [3].

Because of the high morbidity and mortality related to intracerebral hemorrhage, several prognostication scores have been studied, validated and used, the most common of which is the Intracerebral Hemorrhage Score (ICHS) [3]. ICHS is used to predict 30-day mortality rate in patients with hemorrhagic stroke [4], and it has predicted that a score $\geq 3$ is related to poor outcome [5]. The ICH score is very simple in stratifying patients with primary intracerebral hemorrhage and its correlation with surgical outcome proved to be statistically significant, suggesting that high ICH score should have preoperative counseling prior to surgical operation because of poor prognosis [6].

The ICH score was also studied in Asian population confirming that the higher ICH score was associated with higher mortality as in previous findings [7].

The SPAN-100 (Stroke Prognostication using Age and National Institute of Health Stroke Scale (NIHSS)) index [8] on the other hand, is a new prognostication tool used in predicting functional outcome of patients with ischemic stroke. It is the sum of age and baseline NIHSS dichotomized into scores greater or less than 100 , such that scores $\geq 100$ had poorer prognosis.

After being validated in 2012, SPAN-100 index has been used in trials for ischemic stroke. The Chinese Medicine NeuroAid Efficacy on Stroke Recovery (CHIMES) trial that investigated the efficacy of NeuroAid in improving functional outcome following ischemic stroke utilized the SPAN-100 index in evaluating its ability to predict mRS scores at month 3 [8]. Data showed a positive correlation between SPAN-100 index and month-3 mRS ( $p \leq .001)$.

A limitation however, of SPAN-100 index was that after being dichotomized, patients less than 58 years have less than 100 scores even if they were assessed to have maximum scores on NIHSS (42) [9]. SPAN-100 index also suffered from low performance in predicting 3and 12- months outcome of ischemic stroke in Chinese population - an index of 80 could have improve its performance but further statistical evaluation should be applied on each individual [10]. Also, SPAN-100 index has never been used in intracerebral hemorrhages despite the fact that NIHSS is also employed to look into the severity of such stroke cases.

\section{Objective/s}

This study aimed to predict the SPAN index that would correlate with the ICH scores following primary intracerebral hemorrhages. This potential utility maybe used in predicting outcome, benefits, as well as risks of treatment (i.e.surgical evacuation of hematoma) in primary intracerebral hemorrhages.

\section{Methodology}

\section{Research design}

This is a retrospective study that reviewed all admitted cases of intracerebral hemorrhages that utilized ICHS from 2010-2014 in Jose Reyes Memorial Medical Center. From these data, we aimed to calculate the SPAN-100 scores and perform correlational analysis on to which scores correlate to each ICHS item (i.e. ICH scores 0-2 denotes better outcome; ICH score 3-5 denotes poorer outcome). To determine the SPAN score, baseline NIHSS and age were added. The initial ICH score that is defined according to volume of hemorrhage, age, initial Glasgow coma scale (GCS), intraventricular extension and infratentorial level of hemorrhage were also determined.

\section{Study subjects}

Inclusion criteria: All patients admitted in Jose R. Reyes Memorial Medical Center secondary to primary intracerebral hemorrhage from 2010-2014, with determined NIHSS and ICH scores during the first 24 hours upon symptom onset, were included in the study.

Exclusion criteria: Patients who presented with acute intracerebral hemorrhage secondary to trauma, ruptured aneurysm, or arteriovenous malformation, had history of previous stroke (infarct or bleed), and presented to the emergency department $>24$ hours after symptom

Correspondence to: Jose R. Reyes Memorial Medical Center, Rizal Avenue, Sta Cruz, Manila, Philippines, Tel: (02) 711-9491 loc 292, 09228014837; E-mail: cyrusesca@gmail.com

Key words: astrocyte, brain, enriched environment, glia, glutamate

Received: September 14, 2015; Accepted: September 28, 2015; Published: September 30, 2015 
were excluded. Patients who transferred to other hospitals will also be excluded in order ensure availability of records related to initial evaluation.

\section{Measures}

The SPAN-100 index, which combined age (in years) and NIHSS, was originally created for patients with ischemic stroke eligible for intravenous tissue plasminogen activator (tPA). Individuals who scored greater than or equal to 100 designated as SPAN-100-positive patients, had worse outcomes and had high risk of intracerebral hemorrhages, while those whose score was $<100$ who were designated as SPAN-100negative had favorable composite outcomes in 3 months.

This study tested the applicability of SPAN-100 index in patients who had intracerebral hemorrhages. A total of 316 patients were included. A non contrast CT images of the brain, which were readily available from the records of the Radiology Department of JRRMMC was mandatory. All baseline CT scans was obtained with $10-\mathrm{mm}$ slice thickness. The age, NIHSS and the ICH scores were determined along with other baseline characteristics. Two neurologists who were not part of this study were employed to compute for the volume of blood, as part of the ICHS.

The SPAN score, which is the sum of age and NIHSS, were determined and were correlated with the corresponding ICHS. We determined the SPAN score that would correlate to good prognosis (defined as ICHS 0-2) and worse outcome (ICHS 3-5). The mean SPAN score determined was 70 .

\section{Statistical analysis}

To compare the categorical variables, t-test was employed for analysis. The primary analysis was conducted to evaluate the association of a SPAN score of 70 and the outcomes (as dichotomized earlier to ICHS 0-2 and 3-5).

A hypothetical analysis was conducted to compare the different points above 70 (i.e. $75,80,85,90,95,100,105,110)$ that would have a significant outcome. All tests were 2 -tailed, and $p$ values $<0.05$ were considered significant.

\section{Results}

The SPAN index was applied to a total of 316 patients, and the mean score was 70 (range of $68-71$ ).53.5\% of patients had a score of less than 70 , while $46.5 \%$ had a score of greater than or equal to 70 .

Using the score of 70 , statistical analysis showed that there was no significant evidence to say that a score of 70 had worse prognosis (ICHS 3-5), $p=0.7156$. On the contrary, using the original SPAN-100 index showed that a score of greater than or equal to 100 carries 5 times risk (RR 4.99) of having worse prognosis than those with a score of less than 100 .

Further hypothetical sub-analysis to look for a score that can correlate with the dichotomized outcome revealed that a SPAN score of 75 carries a high risk of worse prognosis $(p<0.0001)$. Subsequent analyses with increments of 5 (i.e. 80,85 , etc) have shown the same significant results. Hence, for intracerebral hemorrhages, SPAN-75negative patients had good outcomes while SPAN-75-positive patients had worse prognosis.

The table below (Table 1) shows the baseline characteristics of patients included in this study. $37.3 \%$ of our patients were SPAN75-positve while $62.7 \%$ were SPAN-75-negative. The mean baseline NIHSS was 11 in the SPAN-75-negative and 21 in the SPAN-75-positve patients. The mean age was 49 and 63 for SPAN-75-negative and SPAN75-positive, respectively. Other differences in baseline characteristics are summarized below.

Compared to SPAN-75-positive, SPAN-75-negative patients had significantly lower ICHS $(p<0.0001)$ and lower blood volumes $(p<0.0002)$. This correlated with good prognosis or better outcomes following intracerebral hemorrhage.

\section{Discussion}

Grading scales play an important role in the evaluation and management of patients with acute neurological disorders like in various types of stroke. Clinical practitioners need simple and practical tools when discussing prognosis to patients and their relatives especially after an intracerebral hemorrhage as this has higher propensity to worsen because of hematoma expansion and/or edema. The prediction of a favorable outcome, hence, may be quite challenging. Althoughclinicians have been using the ICHS as a prognostication tool, its utility in the presence of primary infratentorial hemorrhage has not been fully underscored [11].

Factors like age and stroke severity are major determinants of stroke outcomes and while systematically incorporating these prognosticators in practice can be perplexing, the earlier study on SPAN has successfully combined the two. In cases of intracerebral hemorrhages however, this study has been the first to report its utility.

The results above showed that a lower SPAN score (75) corresponds to worse prognosis (ICHS 3-5), as compared in the previous study on ischemic stroke (which utilized the index of 100). This could be attributed to the fact that intracerebral hemorrhages has higher propensity to expand especially in the presence of a poorly controlled hypertension, hence reflecting high scores in the NIHSS. In the context of an inftratentorial hemorrhage where volume is hard to determine because of the size of the area, and where small volumes may present

Table 1. Baseline characteristics between SPAN-75-positive and SPAN-75-negative patients.

\begin{tabular}{|c|c|c|c|c|c|c|}
\hline Variable & \multicolumn{2}{|c|}{ Exposed (Span Score >=75) } & \multicolumn{2}{|c|}{ Unexposed (Span Score <75) } & P-value & Interpretation \\
\hline & \multicolumn{2}{|c|}{$(\mathrm{n}=118)$} & \multicolumn{2}{|c|}{$(n=193)$} & & \\
\hline Age & \multicolumn{2}{|c|}{$63.76 \pm 1.13$} & \multicolumn{2}{|c|}{$49.1 \pm .65$} & 0.8339 & $>0.05$ \\
\hline \multirow[t]{2}{*}{$\operatorname{Sex}(\%)$} & Female $=56$ & Male $=62$ & Female $=55$ & Male $=138$ & & \\
\hline & 47.46 & 52.54 & 28.50 & 71.50 & & \\
\hline NIHSS & \multicolumn{2}{|c|}{$21.23 \pm 1.011$} & \multicolumn{2}{|c|}{$11.36 \pm .427$} & 0.8149 & $>0.05$ \\
\hline SBP & \multicolumn{2}{|c|}{$182.96 \pm 2.26$} & \multicolumn{2}{|c|}{$173.52 \pm 1.84$} & 0.9881 & $>0.05$ \\
\hline Blood volume & \multicolumn{2}{|c|}{$45.8 \pm 3.9$} & \multicolumn{2}{|c|}{$25.1 \pm 2.3$} & 0.0002 & $<0.05$ \\
\hline ICHS & \multicolumn{2}{|c|}{$4.0 \pm 1.2$} & \multicolumn{2}{|c|}{$1.5 \pm 0.6$} & 0.0001 & $<0.05$ \\
\hline Glucose & \multicolumn{2}{|c|}{$115.34 \pm 2.91$} & \multicolumn{2}{|c|}{$118.96 \pm 2.89$} & 0.9076 & $>0.05$ \\
\hline
\end{tabular}


with major alterations in sensorium and worse neurologic deficits (i.e. locked-in syndrome in pontine hemorrhage), the determination of stroke severity (NIHSS) can actually give a better prognostic value rather than the ICHS.

Other than being a simpler tool compared to ICHS, requiring only age and NIHSS, utility of SPAN-75 index can be employed in patients whose neuroimaging are done from other institutions and estimation of volume is hard to compute. In areas also where neuroimaging is unavailable, the use of this index would help clinicians prognosticate their patients especially if patients' history are highly suggestive of intracerebral hemorrhage. Hence, the advantage of SPAN-75 indexes.

Thus, the utility of SPAN-75 index is indeed beneficial and helpful as a prognostication tool in cases of acute intracerebral hemorrhages.

\section{Limitations of the study}

This study had poor control between the time of ictus and the time when CT scan were done. It has been variously studied that CT images done in less than 6 hours upon ictus have higher propensity to expand, and this may have contaminated the above findings. Also, the researchers have no data as to the outcome of patients who were SPAN75-negative and SPAN-75-positive as no follow up were included.

\section{Recommendation}

A follow up study to validate SPAN-75 should be employed.

\section{References}

1. Brault M, Hootman J, Helmick CG, Thesis KA, Armour BS (2009) Prevalence and common causes of disability among adults - United States, 2005. Morb Mortal Wkly Rep 58: 421-426.
2. Samson RD, Barnes CA (2013) Impact of aging brain circuits on cognition. Eur $J$ Neurosci 37: 1903-1915. [Crossref]

3. Camacho EJ, LoPresti MA, Bruce S, Lin D, Abraham ME, et al (2014) The role of age in intracerebral hemorrhage: an intricate relationship. Austin J Cerebrovasc Dis \& Stroke 1: 1022 .

4. Hemphill JC, Bonovich DC, Besmertis L, Manley GT, Johnston SC (2001) The ICH score: a simple, reliable grading scale for intracerebral hemorrhage. Stroke 32: 891897. [Crossref]

5. Godoy DA, Pinero G, Napoli M (2006) Predicting mortality in spontaneous intracerebral hemorrhage: can modification to original score improve the prediction? Stroke 37: 1038-1044.

6. Rashid HU, Amin R, Rahman A, Islam MR, Hossain M, et al. (2013) Correlation between intracerebral hemorrhage score and surgical outcome of spontaneous intracerebral hemorrhage. Bangladesh Med Res Counc Bull 39: 1-5. [Crossref]

7. Sombat M (2013) Predicting mortality rate with ICH score in Thai intracerebral hemorrhage patient. Neurology Asia 18: 131 - 135.

8. Saposnik G, Guzik AK, Reeves M, Ovbiagele B, Johnston SC (2013) Stroke prognostication using age and NIH stroke scale: SPAN-100. Neurology 80: 21-8. [Crossref]

9. Chen CL, Young SH, Gan HH, Singh R, Lao AY, et al. (2013) Chinese medicine neuroaid efficacy on stroke recovery: a double blind, placebo controlled, randomized study. Stroke 44: 2093-100. [Crossref]

10. Pan Y, Jing J, Zhang R, Zhao X, Liu L, et al. (2014) Poor performance of stroke prognostication using age and National Institutes of Health stroke scale-100 to Predict 3- and 12-month Outcomes of Ischemic Stroke in China National Stroke Registry. J Stroke Cerebrovasc Dis 23: 2335-2340. [Crossref]

11. Hemphill JC 3rd, Bonovich DC, Besmertis L, Manley GT, Johnston SC (2001) The ICH score: A simple, reliable scale for intracerebral hemorrhage. Stroke 32: 891-897. [Crossref] 